\title{
Canadian Consensus Conference on Dementia: Two Years Later
}

Can. J. Neurol. Sci. 2001; 28: Suppl. 1 - S1-S2

It is now three years since the Canadian Consensus Conference convened in Montreal to review evidence, reach consensus and make recommendations on a wide variety of diagnostic and management strategies for dementia. The dizzying pace of progress in the whole field of dementia has continued, and our approaches to management, in particular, have continued to evolve.

In recent years the interest in mild cognitive impairment (MCI) has escalated. MCI is the border state between normality and dementia. Many attempts have been made to define this prodromal stage of Alzheimer's disease (AD), and considerable controversy has been generated concerning its exact definition. ${ }^{1,2}$ As consensus slowly emerges, it is evident that MCI, characterized by memory loss but with preservation of other domains of cognition, progresses at a predictable annual rate of $5-15 \%$ to $\mathrm{AD}$. $^{1}$ There is intense interest in the condition, and currently therapeutic trials, which aim to delay progression to dementia, are underway with agents such as estrogens, cholinesterase inhibitors, COX-II inhibitors, and Vitamin E.

Alzheimer's disease has a multifactorial etiology. Genetic factors include predisposing genes (especially the apoE-4 allelic genotype) and rare deterministic genes on chromosomes $1,14,21 .^{3}$ There appears to be a complex interplay among factors such as ischemia, inflammation and excessive beta amyloid production and deposition. A recent review describes these interacting factors. ${ }^{4}$

There have been several advances in therapeutics. A second acetylcholinesterase inhibitor, rivastigmine, has been approved for use in Canada for mild to moderate stages of $\mathrm{AD},{ }^{5,6}$ and a third, galantamine, will be available in the near future. ${ }^{7,8}$ These agents have generally similar properties to donepezil. The results of a multi-centre clinical trial of donepezil in moderate to severe AD have been presented. ${ }^{9}$ This study revealed that donepezil has positive effects on behaviour, performance of daily activities and reduction of caregiver stress well into the later stages of $\mathrm{AD}$. These results were not entirely anticipated, as many would have predicted from the cholinergic hypothesis that such agents would have less, and not more, benefits in later disease, as further degeneration of cholinergic neurons occurred. This study paves the way for broader indications for cholinesterase inhibitors, and we may see these agents used for apathy and other behavioural problems in more advanced cases of dementia.

Published case series have suggested that donepezil is helpful in managing the hallucinations, delusions, cognitive and behavioural symptoms in dementia with Lewy bodies. ${ }^{10}$ Subsequently, a large randomized placebo controlled trial of rivastigmine in dementia with Lewy bodies has confirmed the benefit of cholinesterase inhibitors in this disorder. These findings were presented in abstract at the World Congress of
Alzheimer's Disease in July 2000 and are currently also in press. There have been advances too in the management of difficult behaviours (behavioural and psychological symptoms of dementia - BPSD). Two large randomized controlled trials have demonstrated the efficacy of risperidone, an atypical neuroleptic agent, in the management of aggression and other behavioural problems in dementia. ${ }^{11,12}$ Furthermore, in one of these studies ${ }^{11}$ risperidone was clearly superior to haloperidol, a frequently prescribed traditional neuroleptic agent. A clinical trial of olanzepine has revealed that this agent is also beneficial and safe in BPSD $^{13}$ and it is probable that quetiapine, another atypical neuroleptic agent will also prove efficacious in this setting. Despite their cost, the significantly lower risk of extrapyramidal side effects, particularly parkinsonism and tardive dyskinesia, together with their proven efficacy are leading to their emergence as first line agents in BPSD.

Regardless of these advances in therapy, the diagnosis of dementia still depends heavily upon a careful history and gathering of information from corroborative sources. History, together with physical, neurological and cognitive examinations, a limited number of laboratory investigations and selective neuroimaging continue to constitute the mainstay of appropriate diagnosis. Support of the caregiver, recruitment of community supports and enrollment in advocacy organizations such as the Alzheimer's Society will always be the cornerstone of therapy. While Alzheimer's and other dementias continue to be terrifying diseases, they are far less so than in the past, as the exponential growth of knowledge leads us to better understanding and constantly improving therapies.

C. Patterson, McMaster University, Hamilton, $O N$ S. Gauthier, McGill University, Toronto, ON Co-chairs of the Canadian Consensus Conference on Dementia

\section{REFERENCES}

1. Petersen RC, Smith GE, Waring SC. Mild cognitive impairment. Clinical characterization and outcome. Arch Neurol. 1999; 56: 303-308.

2. Ritchie K, Touchon J. Mild cognitive impairment: conceptual basis and current nosological status. Lancet 2000; 355: 225-228.

3. Blacker D, Tanzi RE. The genetics of Alzheimer disease. Arch Neurol 1998; 55: 294-296.

4. Munoz DG, Feldman H. Causes of Alzheimer's disease. CMAJ 2000; $162:$ 65-72

5. Corey-Bloom J, Anand R, Vach J for the ENA 713 B352 Study Group. A randomized trial evaluating the efficacy and safety of ENA 713 (rivastigmine tartrate), a new acetylcholinesterase inhibitor, in patients with mild to moderately severe Alzheimer's disease. Int J Geriatric Psychopharmacol 1998; 1: 55-65.

6. Rosler M, Anand R, Cicin-Sain A, et al. Efficacy and safety of rivastigmine in patients with Alzheimer's Disease: international randomised controlled trial. Br Med J 1999; 318 (7184):633-638. 
7. Raskind MA, Peskind ER, Wessel T, Yuan W, and the Galantamine USA-1 Study Group. Galantamine in AD. A 6-month randomized, placebo-controlled trial with a 6-month extension. Neurology 2000; 54: 2261-2268.

8. Tariot PN, Solomon PR, Morris JC, Kershaw P, Lilienfeld S, Ding $\mathrm{C}$, and the Galantamine USA-10 Study Group. A 5-month randomized, placebo-controlled trial of galantamine in AD. Neurology 2000; 54: 2269-2276.

9. Feldman H, Gauthier S, Hecker J, Vellas B, Subbiah P, Whalen E, and the Donepezil MSAD Study Group. Benefits of donepezil on global function, behaviour, cognition, and ADLs in patients with moderate-to-severe Alzheimer's disease. Neurology 2000; 54(3); A469.
10. Shea C, Macknight C, Rockwood K. Donepezil for treatment of dementia with Lewy bodies: a case series of nine patient. Int Psychogeriatr 1998; 10(3): 229-238.

11. De Deyn, PP, Rabheru K, Rasmussen A, et al. A randomized trial of risperidone, placebo, and haloperidol for behavioural symptoms of dementia. Neurology 1999; 53:946-955.

12. Katz IR, Jeste DV, Mintzer JE, et al. Comparison of risperidone and placebo for psychosis and behavioural disturbances associated with dementia: A randomized, double-blind trial. J Clin Psychiatry 1999; 60:107-115.

13. Defilippi JL, Crismon ML. Antipsychotic agents in patients with dementia. Pharmacotherapy 2000; 20(1): 23-33. 